\title{
Overview on Modeling and Design of Stratospheric Mobile Cellular System
}

\author{
Sultan Aljahdali \\ Dept. of Computer Science, College of \\ Computers and Information Technology, \\ Taif University \\ Taif, Kingdom of Saudi Arabia \\ e-mail: aljahdali@tu.edu.sa
}

\author{
Mostafa Nofal \\ Dept. of Computer Engineering, College \\ of Computers and Information \\ Technology, Taif University \\ Taif, Kingdom of Saudi Arabia
}

\author{
Yasser Albagory* \\ College of Computers and Information \\ Technology, Taif University, Taif, \\ Kingdom of Saudi Arabia \\ *e-mail: y.albagory@tu.edu.sa
}

\begin{abstract}
In this paper, cellular communications from Stratospheric Platforms (SPs) is studied and the footprint analysis and design are demonstrated. In the analysis part two coverage schemes are discussed; flat-earth and realearth models and the cell footprint is determined in each case. The flat-earth provides simple footprint equations describing the cell dimensions especially for cells of higher elevation angles while more accurate coverage equations can be obtained from the real-earth scheme which well determine the geometry of the cells of lower elevation angles. The design of a cellular system using the proposed coverage models is then demonstrated through a procedure that determines the cells locations and dimensions on the ground according. The procedure takes into consideration the cell broadening when going outwardly from the central cell to the outer cells and an update for the conventional terrestrial equations must be performed to properly locate the cells. The convergence in calculating the cell parameters have been shown to be fast and reach the final values after few updating cycles.
\end{abstract}

Keywords-Stratospheric platforms, mobile communications,

directional antennas, phased antenna arrays.

\section{INTRODUCTION}

In recent years, Stratospheric Platforms (SPs) has a considerable interest in broadband mobile communications. These platforms are located 17-20 km high above the earth's surface and can provide variety of applications. The demand for SPs has increased as it has most advantages of the conventional systems such as terrestrial mobile radio and satellite systems while eliminating most of their problems as shown by many studies [1-3]. It is expected that SPs will play an important role in the future of wireless communications beyond $3 \mathrm{G}$ systems [3]. One of the important issues in SPs communications is to provide higher system capacity and this can be obtained through utilizing the frequency reuse over the covered area. According to the reuse factor and the needed carrier-to-interference ratio (CIR), the cellular system will be constructed defining each cell location and dimensions. In the terrestrial mobile system, the cells are defined by the hexagonal shape while in the case of SPs, the cell footprint has an ellipsoidal shape [2] which has a major and minor axes defining its dimensions. The SPs ground cells can be formed using either spot-beam antennas or phased arrays and the cell boundary is well defined by the beam pattern of the used antenna. A difficulty in deploying this cellular system is that the cell footprint increases as the elevation angle decreases which requires some modification in pointing the beams of these cells. Another point worth noting is that most of the radio coverage studies [2] for SPs had approximated the earth as a flat surface as the platform altitude is much smaller than the earth's radius, while for large coverage areas we need an accurate cell dimensioning taking into considerations the earth curvature. Therefore this paper is devoted to overview the main aspects of cell layout and design for SPs. The paper is arranged as follows: in section II the flat-earth approximation is depicted and discussed while section III discusses the realearth coverage model. Section IV reviews the design aspects of SPs cellular system. Section V analyzes the terrestrial cellular design while in section VI the SPs cellular-layout procedure is introduced and the study of convergence of calculation of cell parameters is performed. Finally section VII gives some concluding remarks.

\section{Flat-EARTH COVERAGE Model}

The SPs wireless communication system may utilize the directional antennas as well as antenna arrays to construct cellular footprint [4]. Directional antennas may be in the form of parabolic reflectors, horn antennas, or any other suitable antenna that gives the desired directional pattern. The use of directional antennas has some advantages such as its practical availability and simplicity. The ground cells footprints can be also formed by directing a beam using phased arrays which has a widespread use. From the wind profile studies [3], the SPs station is located at an altitude about $20 \mathrm{~km}$ high, which is very small compared with the earth's radius, therefore the earth is approximated as a flat surface as shown in Fig. 1. 


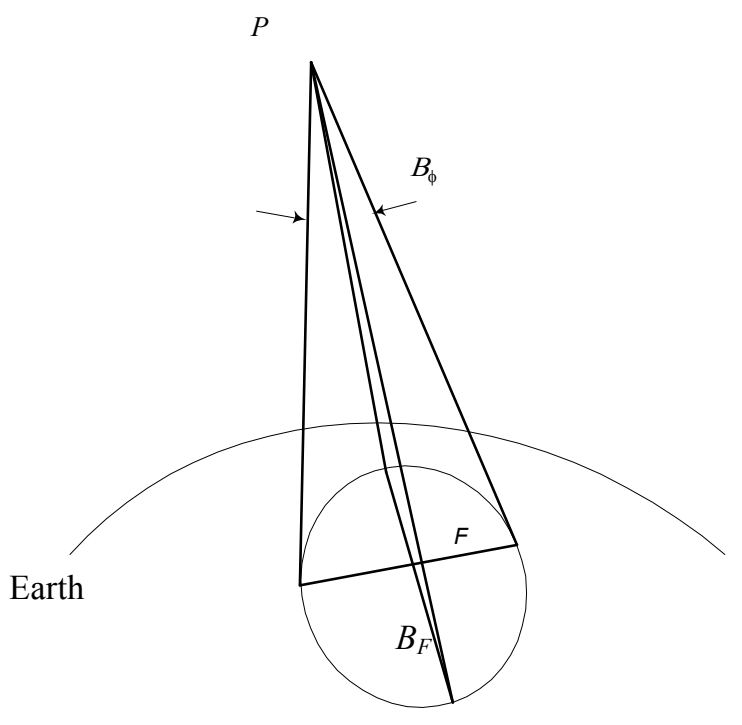

Figure 1: SP cell footprint

In this figure, the footprint of a beam formed onboard the $\mathrm{SP}$ is shown. The cell as depicted in Fig. 1 is defined by the coverage beam that has a direction of $\theta_{o}$ as shown in Fig. 2 and cross section beamwidths of $B_{\theta}$ and $B_{\phi}$, and the footprint on the ground will be approximately an ellipse that has a major axis $b_{F}$ which can be defined as

$$
b_{F}=h\left(\tan \left(\theta_{o}+\frac{B_{\theta}}{2}\right)-\tan \left(\theta_{o}-\frac{B_{\theta}}{2}\right)\right)
$$

where the subscript $F$ stands for flat ground approximation and $h$ is the platform altitude in $\mathrm{km}$. The cell center, $C$, is located by an angle from the platform given by

$$
\theta_{c}=\tan ^{-1}\left(\tan \left(\theta_{o}-\frac{B_{\theta}}{2}\right)+\frac{b_{F}}{2 h}\right)
$$

and if the cell minor axis is denoted by $a_{F}$, it can be given by

$$
a_{F}=2 h \sec \left(\theta_{\mathrm{c}}\right) \tan \left(\frac{B_{\phi}}{2}\right)
$$

The minor and major axes will define the cell shape and this assumption can be used for smaller and moderate SP coverage areas.

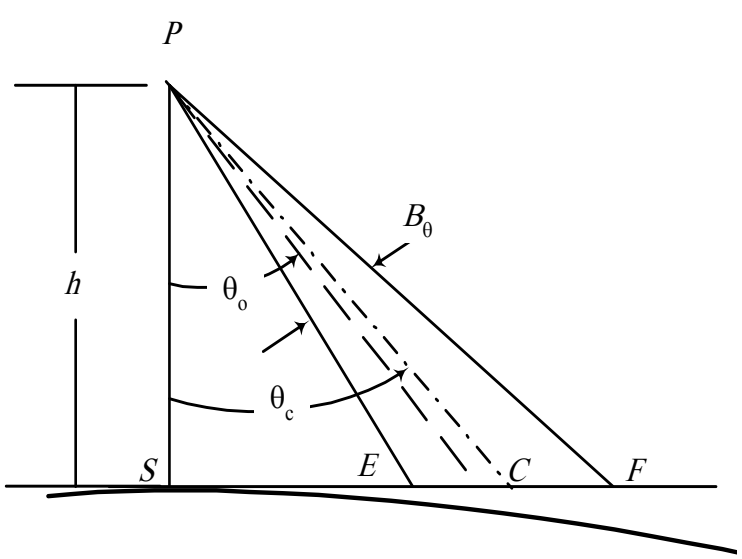

Figure 2: Flat-Earth approximation geometry

\section{REAL-EARTH COVERAGE MOdell}

For larger covered areas, one must take into account the spherical property of the earth. In [4], the earth curvature is taken into consideration which good predicts the cell footprint. A side view is shown in Fig. 3, which depicts the geometry used to define the cell parameters.

In this figure, the major axis will be the arc on the earth's surface between the two points $\mathrm{E}$ and $\mathrm{F}$ passing through $\mathrm{C}$ and is given by:

$$
\begin{aligned}
& b_{C}=R\left(\sin ^{-1}\left(\left(1+\frac{h}{R}\right) \sin \left(\theta_{o}+\frac{B_{\theta}}{2}\right)\right)\right. \\
& \left.-\sin ^{-1}\left(\left(1+\frac{h}{R}\right) \sin \left(\theta_{o}-\frac{B_{\theta}}{2}\right)\right)-B_{\theta}\right)
\end{aligned}
$$

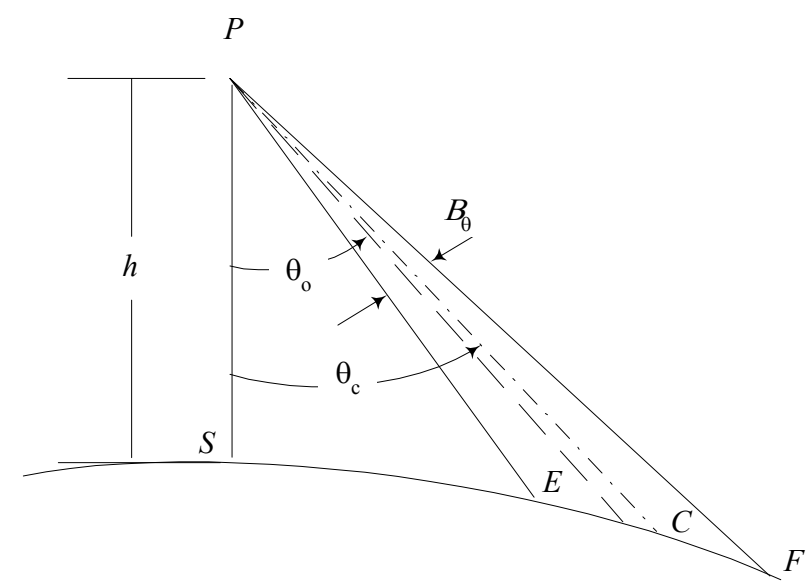

Figure 3: Curved earth coverage geometry

where the subscript $\mathrm{c}$ stands for curved-earth and $R$ is the earth's radius and in this case the value of $\theta_{c}$ will be 


$$
\theta_{c}=\tan ^{-1}\left\{\frac{\tan \left(\gamma_{o}\right)}{2\left(1+\frac{h}{R}\right) /\left(\cos \left(\gamma_{1}\right)+\cos \left(\gamma_{2}\right)\right)-1}\right\}
$$

where the angles $\gamma_{1}$ and $\gamma_{2}$ are defined as

$$
\gamma_{1}=\sin ^{-1}\left(\left(1+\frac{h}{R}\right) \sin \left(\theta_{o}-\frac{B_{\theta}}{2}\right)\right)-\theta_{o}+\frac{B_{\theta}}{2}
$$

and

$$
\gamma_{2}=\sin ^{-1}\left(\left(1+\frac{h}{R}\right) \sin \left(\theta_{o}+\frac{B_{\theta}}{2}\right)\right)-\theta_{o}-\frac{B_{\theta}}{2}
$$

and the cell center has a ground central angle given by

$$
\gamma_{o}=\frac{1}{2}\left(\gamma_{1}+\gamma_{2}\right)
$$

The cell minor axis, $a_{C}$, is also given by:

$$
a_{C}=2 h \sec \left(\theta_{c}\right) \tan \left(\frac{B_{\phi}}{2}\right)
$$

which can be also expressed by:

$$
\begin{aligned}
& a_{C}=2 R \tan \left(\frac{B_{\phi}}{2}\right)\left(\left(1+\frac{h}{R}-\frac{1}{2}\left(\cos \left(\gamma_{1}\right)+\cos \left(\gamma_{2}\right)\right)\right)^{2}\right. \\
& \left.+\frac{1}{4}\left(\cos \left(\gamma_{1}\right)+\cos \left(\gamma_{2}\right)\right)^{2} \tan ^{2}\left(\gamma_{o}\right)\right)^{1 / 2}
\end{aligned}
$$

Both the major and minor axes show the effect of the earth curvature and it is appeared by the inclusion of the earth radius and ground angles in the equations.

\section{SPs Radio Coverage Design Issues}

When using the same type of antenna in all formed cells, the uniformity of the cellular structure is important especially when we plan a network containing a number of SPs [5]. In the following sections, we assume that we have directional antennas or antenna arrays that give the desired parameters and we discuss how the cells can be arranged on the ground to have most uniform cellular coverage.

In terrestrial cellular systems, the cell is approximated mathematically as a hexagon of certain corner radius which simplifies the analysis, but in the case of SP system, the cell boundary will be of circular or elliptical shape affecting the construction of the cellular layout. In [5], some adjustments are performed for the calculated terrestrial system geometry to have the proper SP cellular structure and these adjustments will be function of the cells locations. Therefore in the next sections, we recall the hexagonal cellular geometry and the parameters of the cells in the terrestrial system and then modifying these parameters to suit the SP system.

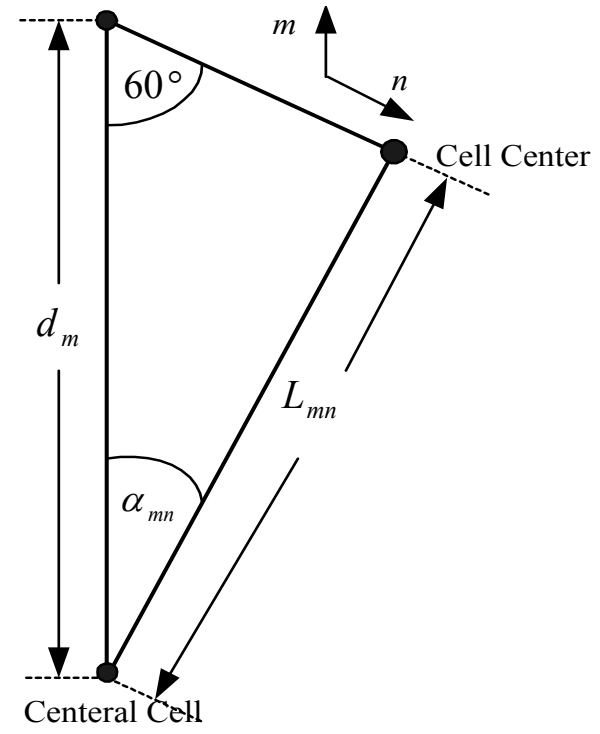

Figure 4: Coordinate system for cell location determination

\section{TERrestrial Cellular Structure}

Due to the symmetry of the hexagon, hexagonal cellular layout geometry is divided into six homogenous sectors and for any sector we use the integers $m$ and $n$ to define the cell location or coordinates as shown in Fig. 4. In general each cell has a separating distance from the center of the central cell given by $L_{m n}$ and an azimuth angle, $\alpha_{m n}$, measured from the vertical axis, $\mathrm{m}$, to the line connecting the center of that cell with the center of the central cell. From the geometry of this coordinate system we can define $L_{m n}$ as [5]:

$$
L_{m n}=d_{1} \sqrt{m^{2}+n^{2}-m n}
$$

where $d_{1}=r_{o} \sqrt{3}$ and $r_{o}$ is the central cell radius.

Also $\alpha_{m}$ can be given by [5]:

$$
\alpha_{m n}=\cos ^{-1}\left(\frac{2 m-n}{2 \sqrt{m^{2}-m n+n^{2}}}\right)
$$

Figures 5 and 6 demonstrate some of the cell central distances and azimuth angles respectively in the first sector. For the tier of order $m$, we find that $n$ will take the values 0,1 , $2, \ldots m$. 


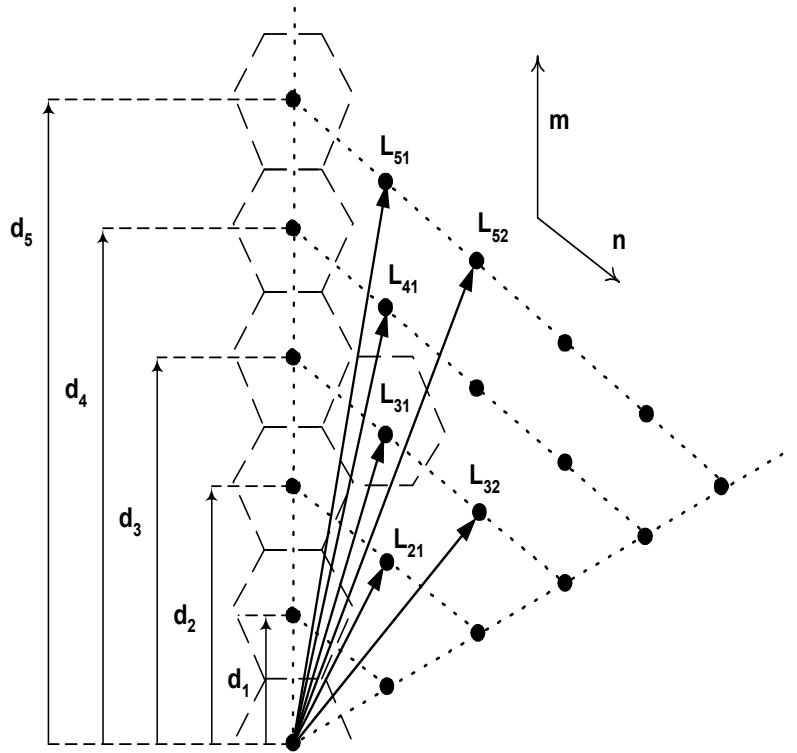

Figure 5: Central distance $L_{m n}$ and radial distance $d_{m}$ for some cells located in the first sector cell

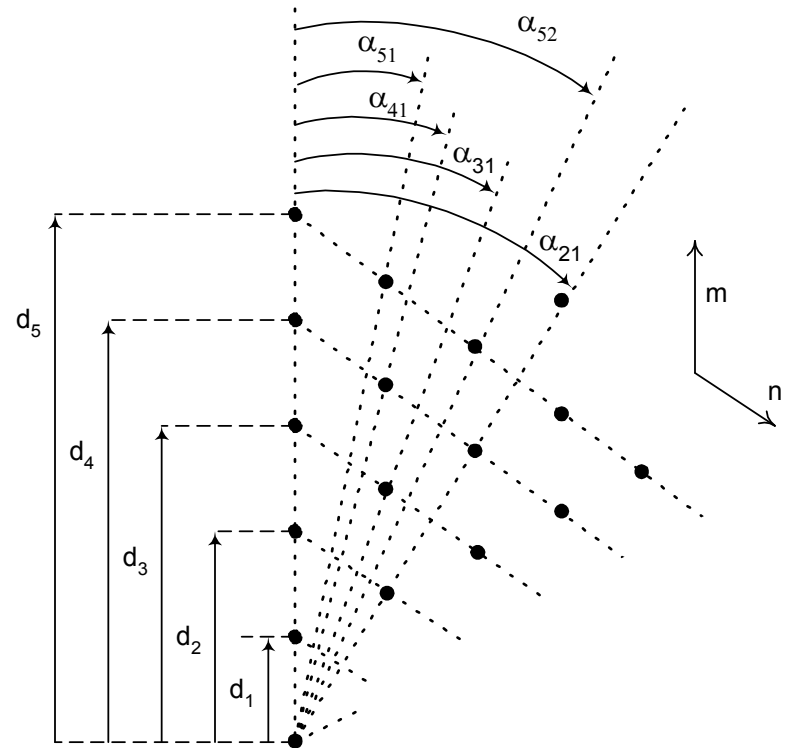

Figure 6: Central azimuth angle $\alpha_{m n}$ for some cells located in the first sector

\section{SPS CELLULAR SYSTEM DEPLOYMENT}

Designing SPs cellular system means defining each cell location and the corresponding antenna parameters used in the radio coverage such as beamwidth, gain, bandwidth and the pointing angles or the beams directions. An algorithm is proposed that generates the antenna pointing angles taking into consideration the uniformity of the radio coverage such as minimizing the coverage gaps and overlap. The algorithm proceeds outwardly from the central cell and starts with the value of the central cell radius $r_{o}$, then we calculate the first tier cells using the conventional terrestrial relations discussed in the last section and make some corrections due to the cell flattening. For the other outer tiers we update the central distances by the new expected major axis of the cell. The algorithm calculates the cells on the vertical axes (i.e. the cells having $\alpha_{m}=0$ degree). The other cells locations in the outer tiers that having azimuth angles other than zero are determined by taking the ratio of the corresponding cells radial distance $d_{m}$ with $L_{m 0}$ calculated from Eq. 11 which is then multiplied by the distance $L_{m n}$ to find the cell location with its azimuth $\alpha_{m n}$ given in Eq. 12. This algorithm can be summarized with its relations in the following points:

1- From the teletraffic information find the central cell radius $r_{o}$ and the needed antenna beamwidths $B_{\theta}$ and $B_{\phi}$.

2- Find the initial radial distance $d_{1}=r_{o} \sqrt{3}$,

3- Find $\theta_{o}=\tan ^{-1}\left(\frac{d_{1}}{h}\right)$ and $b_{c 1}$,

4- Update the radial distance $d_{1}$ with $d_{1}=\frac{\sqrt{3}}{2}\left(r_{o}+\frac{b_{c 1}}{2}\right)$

5- Update $\theta_{o}$ with $\theta_{o}=\tan ^{-1}\left(\frac{d_{1}}{h}\right)$

6- Determine the final values of $a_{c 1}$ and $b_{c 1}$

For the other outer tiers, the algorithm proceeds outwardly and if the cells are located on the sectors edges (i.e. their azimuth angles are $0^{\circ}, 60^{\circ}, 120^{\circ}$, etc.), then the cells locations are calculated as follows:

1- Start with $d_{m}=d_{m-1}+\frac{\sqrt{3}}{2} b_{c m-1}$, where $m$ is the order of the tier.

2- Find $\theta_{o}$ and $b_{c m}$,

3- Update $d_{1}$ with $d_{m}=d_{m-1}+\frac{\sqrt{3}}{4}\left(b_{c m}+b_{c m-1}\right)$

4- Calculate the final values of $\theta_{o}, b_{c m}, \theta_{c}$, and $a_{c m}$.

5- For other non $60^{\circ}$-axes cells we find the ratio $d_{m} / L_{m 0}$, where $L_{m 0}$ is calculated from Eq. 11, with $n=0$ and $m$ is the order of the tier, then multiply this ratio by $L_{m n}$ which gives the needed cell radial distance:

$$
L_{m n}^{\prime}=\frac{d_{m}}{L_{m 0}} L_{m n}
$$


6- From the value of $L_{m n}^{\prime}$ calculate $\theta_{o}=\tan ^{-1}\left(\frac{L_{m n}^{\prime}}{h}\right)$, $b_{c m}, \theta_{c}$ and $a_{c m}$

The updating steps in the algorithm may be repeated several times to reach the final cells locations. Figures 7-a and 7-b depict the convergence in calculations of the cell distance $d_{m}$ for the first, second and third tiers for an array $10 \times 10$ elements and spot-beam antennas of $10^{\circ}$ beamwidth. In both figures, the final value of $d_{m}$ may be reached in one updating cycle especially for the internal tiers while it may need few updates for the outer ones. The use of antenna arrays may require slightly more update steps than that for the spot beam case and this is may be due that the array pattern becomes wider for the outward cells in the outer tiers than for the inner ones.

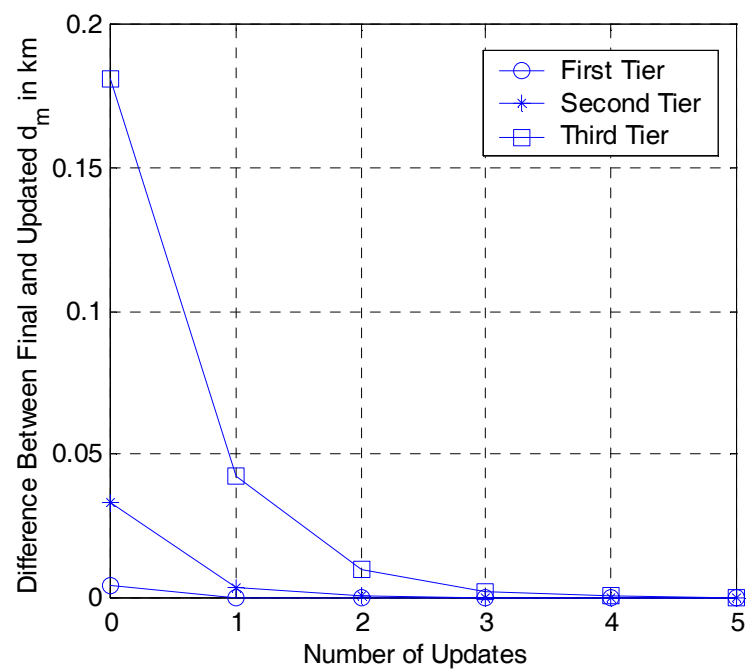

Figure 7-a: Convergence of the cells distance $d_{m}$ for the first three tiers constructed by an antenna array $10 \times 10$ elements.

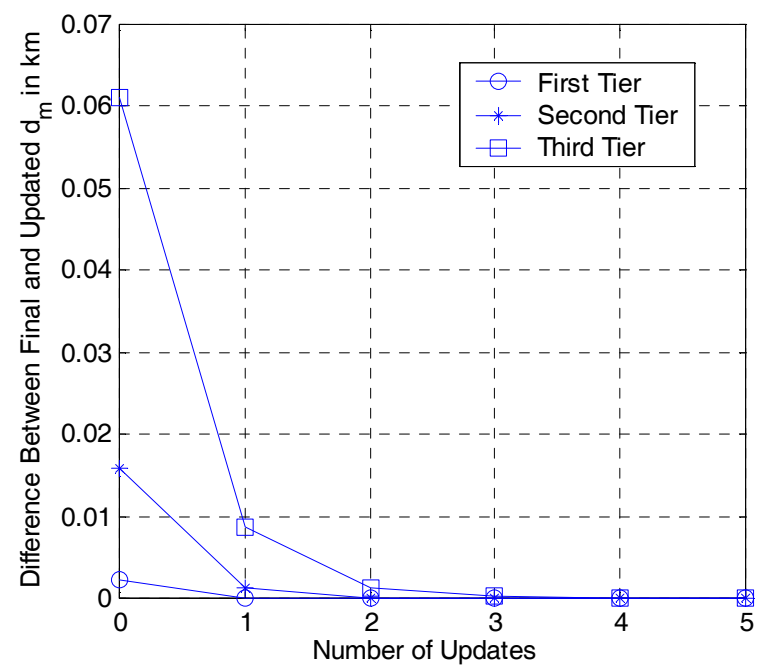

Figure 7-b: Convergence of the cells distance $d_{m}$ for the first three tiers constructed by spot-beam antennas of $10.16^{\circ}$ beamwidth.

\section{CONCLUSION}

Cellular communications from Stratospheric Platforms (SPs) has been discussed and the geometry of the coverage footprint is reviewed and demonstrated. Two coverage configurations are discussed depending on the approximation of the earth's surface; flat-earth and curved-earth coverage. In flat-earth model there are simple footprint equations describing the cell dimensions especially for cells of higher elevation angles while more accurate coverage equations can be obtained from the curved-earth scheme which takes into consideration the curvature of the earth and well determine the geometry of the cells of low elevation angles. The two models can be used for cell dimensioning where one can utilize flat earth model for cells of higher elevation angles while for wider coverage beams and lower elevation angles, the other curved-earth model can be utilized. Dimensioning the cells will be very important in the next step of the cellular design which introduced through an algorithm that determines the cells locations and dimensions on the ground according to the teletraffic information. The algorithm takes into considerations the cell broadening when increasing its going outwardly from the central cell to the outer tiers of low-elevation angle cells. A convergence study for the steady cell parameters has shown that we can reach these values with a few updating cycles and the number of update cycles for antenna arrays is very slightly higher than that of the spot-beam antennas which may be due to the broadening occurred in the outer cells in the case of antenna arrays.

\section{REFERENCES}

[1] [G. M. Djuknic, J. Freidenfelds, and Y. Okunev, "Establishing Wireless Communications Services via High Altitude Platforms: A Concept Whose Time Has Come?," IEEE Commun. Mag., vol. 35, no. 9, Sept. 1997, pp. 128-35.

[2] B. El-Jabu and S. Steele, "Cellular Communications Using Aerial Platforms," IEEE Trans. Vehic. Tech., vol. 50, no. 3, May 2001, pp. 686-700.

[3] S. Karapantazis and F. N. Pavlidou "Broadband Communications via High-Altitude Platforms: a Survey," IEEE Communications Surveys \& Tutorials, first quarter 2005 , volume 7 , no. 1.

[4] Yasser Albagory Moawad Dessouky, Hamdy Sharshar, "Geometrical Analysis of High Altitude Platforms Cellular Footprint," Progress In Electromagnetics Research, PIER 67,pp. 263-274, 2007.

[5] Yasser Albagory, Moawad Dessouky, Hamdy Sharshar, "Design of High Altitude Platforms Cellular Communications," Progress In Electromagnetics Research, PIER 67, pp. 251-261, 2007. 